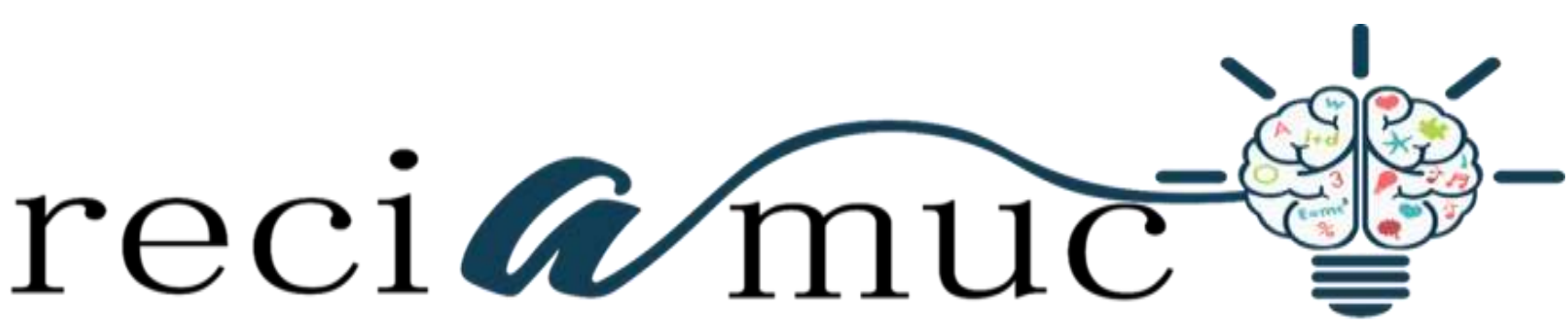

Revista cientifica de investigación actualización del mundo de las ciencias

Clara de las Mercedes Razo Ascazubi a ; Jeanette Lorena Moscoso Córdova ${ }^{\text {b; }}$ Patricia Geraldina López Fraga ${ }^{c}$

La Contabilidad Internacional: incluye visión histórica, paradigma y teorías

International Accounting: includes historical vision, paradigm and theories

Revista Científica de Investigación actualización del mundo de las Ciencias. Vol. 3 núm., 3, julio, ISSN: 2588-0748, 2018, pp. 921-952

DOI: $10.26820 /$ reciamuc/3.(3).julio.2019.921-952

\section{URL: http://reciamuc.com/index.php/RECIAMUC/article/view/310}

Código UNESCO: 5303 Contabilidad Económica

Tipo de Investigación: Artículo de Revisión

(C) RECIAMUC; Editorial Saberes del Conocimiento, 2019

Recibido: 28/04/2019

Aceptado: 19/05/2019

Publicado: 01/07/2019

Correspondencia: patricia.lopez@utc.edu.ec

a. Magister en Gestión Bancaria y Financiera; Especialista en Gerencia de Administración Financiera; Diploma Superior en Negocios Bancarios; Ingeniera en Finanzas, Contadora Publica - Auditora; Universidad Técnica de Cotopaxi; Latacunga, Ecuador; clara.razo@utc.edu.ec

b. Magister en Tributación y Derecho Empresarial; Ingeniera en Contabilidad y Auditoría CPA; Universidad Técnica de Cotopaxi; Latacunga, Ecuador; jeanette.moscoso@utc.edu.ec

c. Magister en Ciencias de la Educación Mención en Gestión Educativa y Desarrollo Social; Doctora en Contabilidad y Auditoria; Licenciada en Contabilidad y Auditoria Contadora Publica; Universidad Técnica de Cotopaxi; Latacunga, Ecuador; patricia.lopez@utc.edu.ec 


\section{La Contabilidad Internacional: incluye visión histórica, paradigma y teorías}

Vol. 3, núm. 3., (2019)

Clara de las Mercedes Razo Ascazubi; Jeanette Lorena Moscoso Córdova; Patricia Geraldina López Fraga

\section{RESUMEN}

El objetivo de la presente investigación busca disertar sobre la contabilidad internacional desde una visión histórica general, paradigmas y teorías, con incidencia en la ciencia contable, a los fines de abordar los planteamientos investigativos desde la Teoría Crítica de la Contabilidad, haciendo énfasis en los aportes de los investigadores contables, apoyados metodológicamente en revisión de literatura especializada. En este sentido, podemos indicar sobre los intentos recientes por re-conceptualizar el papel de la contabilidad internacional en las organizaciones y la sociedad. El concepto de contabilidad internacional, sin embargo, no se tiene desarrollado dentro de una sola tradición teórica, más bien surge de varias tradiciones sociológicas o corrientes filosóficas como el idealismo, realismo, positivismo e interpretacionismo, cada una sugiriendo una perspectiva distinta sobre los fenómenos y abriendo nuevas áreas para la investigación. Este artículo revisa perspectivas teóricas, ubicando las investigaciones existentes sobre contabilidad internacional dentro de ellas y sugiere algunas áreas para futuras investigaciones.

Palabras Claves: Contabilidad Internacional; Corrientes Filosóficas; Sociología Contable; Paradigmas Contables; Historia Contable; Ciencias Contables. 


\title{
La Contabilidad Internacional: incluye visión histórica, paradigma y teorías
}

Vol. 3, núm. 3., (2019)

Clara de las Mercedes Razo Ascazubi; Jeanette Lorena Moscoso Córdova; Patricia Geraldina

López Fraga

\begin{abstract}
The objective of this research is to discuss international accounting from a general historical perspective, paradigms and theories, with an impact on accounting science, in order to address the research approaches from the Critical Theory of Accounting, with emphasis on contributions of the accounting researchers, methodologically supported in review of specialized literature. In this sense, we can indicate recent attempts to re-conceptualize the role of international accounting in organizations and society. The concept of international accounting, however, has not been developed within a single theoretical tradition, rather it arises from various sociological traditions or philosophical currents such as idealism, realism, positivism and interpretationism, each suggesting a different perspective on the phenomena and opening new areas for research. This article reviews theoretical perspectives, locating existing research on international accounting within them and suggests some areas for future research.
\end{abstract}

Key Words: International Accounting; Philosophical Currents; Accounting Sociology; Accounting Paradigms; Accounting History; Accounting Sciences. 


\section{La Contabilidad Internacional: incluye visión histórica, paradigma y teorías}

Vol. 3, núm. 3., (2019)

Clara de las Mercedes Razo Ascazubi; Jeanette Lorena Moscoso Córdova; Patricia Geraldina López Fraga

\section{Introducción.}

El presente trabajo relacionado para mostrar el concepto de contabilidad internacional en las organizaciones desde una mirada filosófica, sociológica e histórica, desde cuatros aristas filosóficas que desarrollaremos a lo largo del presente artículo.

En la literatura reciente ha habido intentos de reconceptualizar el papel de la contabilidad internacional. Esto ha sido ocasionado por el fracaso empírico de la Eficiencia, la Teoría del Mercado y la Teoría de la contingencia, es decir, los paradigmas tradicionales.

Para Johnson (1983) proporcionar los fundamentos para el desarrollo de la contabilidad con técnicas y sistemas, así como llamadas explícitas a considerar la contabilidad en su organización y contexto social. Según Cooper et al. (1981), el resultado ha sido el desarrollo de una serie de interesantes enfoques de contabilidad incluyendo los mercados y un marco de jerarquía, la contabilidad en las anarquías organizadas y un enfoque de economía política

Una de las sugerencias más frecuentes, aunque la terminología utilizada no ha sido consistente es que la contabilidad puede ser útil desde lo internacional. Estas sugerencias van desde la de Cooper (1980) afirmación de que "la contabilidad puede ser vista como una medios de sustento y legitimación de la corriente de arreglos sociales, económicos y políticos" y Tinker et al. (1982) en la caracterización de la contabilida como "ideología", y la noción de que la contabilidad sirve de papel "constitucional" en las organizaciones. El enfoque común de estos documentos es la capacidad de la contabilidad, como conjunto de creencias y técnicas, para vincular acciones y valores, es decir, hacer acciones legítimas. 


\section{La Contabilidad Internacional: incluye visión histórica, paradigma y teorías}

Vol. 3, núm. 3., (2019)

Clara de las Mercedes Razo Ascazubi; Jeanette Lorena Moscoso Córdova; Patricia Geraldina López Fraga

\section{Desarrollo.}

Aspectos históricos de la contabilidad general

La contabilidad es una acción anticuada como la propia humanidad. En efecto, prácticamente desde la aparición del hombre, incluso antes de aparecer la escritura, los imperios comenzaron a sentir la necesidad de controlar cuentas, registrar, guardar información y dejar constancia de datos relativos a su vida económica y a su patrimonio, como por ejemplo, de los bienes, la caza de animales, su consumo y los que mantenía para la cría; así mismo, los bienes que almacenaba para futura venta o consumo; los bienes que prestaba o enajenaba; los bienes que daba en administración a otros, etcétera.

Con relación a ello, existen diferentes investigaciones como las de Denise SchmandtBesserat y, sobre todo, las de Hans Nissen, Peter Damerow y Robert Englund (1990), ellos fueron arqueólogos e historiadores de la antigua Mesopotamia.

Los mismos, permitieron afirmar, que los primeros documentos escritos que se conocen, constituidos por millares de tablillas de arcilla con inscripciones en caracteres protocuneiformes, elaboradas hace más de 5.000 años, contienen tan sólo números y cuentas, sin textos ni palabras.

Este hecho hace inferir de los eruditos citados que la escritura debió surgir, hacia el año 3300 a.J., para beneficiarse registro, y, de esta forma, dejar constancia de sus cuentas. En consecuencia, lo que motivó el surgimiento de la escritura, fue la necesidad de recoger y conservar las cuentas producto de los procesos productivos y administrativos de la época. 


\section{La Contabilidad Internacional: incluye visión histórica, paradigma y teorías}

Vol. 3, núm. 3., (2019)

Clara de las Mercedes Razo Ascazubi; Jeanette Lorena Moscoso Córdova; Patricia Geraldina López Fraga

Desde esos primeros tiempos, las actividades de registro contable se han venido sucediendo ininterrumpidamente, aunque sólo a partir de los siglos XIV y XV se conservan testimonios escritos con un carácter regular y relativamente abundante.

Los datos contenidos en la documentación contable, aun la más antigua y, corresponde a elementos netamente cuantitativos y exactos, que luego con la modernidad aparece las contabilidades empleando la partida doble, en las que los datos se presentan formando un todo coherente, completo e íntimamente interrelacionado.

En éste orden de ideas, los autores citados al inicio e investigadores del tema, su estudio e interpretación favorece proyectar una nueva forma de observar los hechos históricos que, a falta de ellos, habían sido explicados de manera errónea o insuficiente.

La experiencia del Imperio Español en materia de análisis histórico-contable

Por tal motivo, podemos expresar que se podrían encontrar muestras de esta nueva luz proporcionada por el análisis histórico-contable, por ejemplo, el estudio del Imperio español de los siglos XVI y XVII la historiografía mundial ha procurado a concentrar sus esfuerzos en elementos relacionados con el poder militar y político que a España le proporcionó la inmensa riqueza en metales preciosos procedentes de las Indias.

Sin embargo, la investigación moderna del aparato contable y organizativo de la Real Hacienda castellana ha manifiesto y llamado la atención sobre el relevante papel jugado en todo este contexto por la formidable organización administrativa del Imperio, creada totalmente ex - 


\section{La Contabilidad Internacional: incluye visión histórica, paradigma y teorías}

Vol. 3, núm. 3., (2019)

Clara de las Mercedes Razo Ascazubi; Jeanette Lorena Moscoso Córdova; Patricia Geraldina López Fraga

novo, sin precedentes de ninguna clase, y que luego sirvió de modelo a todos los sistemas de dominio colonial que siguieron al español.

De este modo, la investigación histórico-contable ha colocado en el primer plano de la investigación histórica a la organización de la administración castellana en ese tiempo, revelándola como uno de los factores más significativos para conocer con profundidad y explicar la evolución y vicisitudes del Imperio.

De igual modo, la investigación histórico-contable ha mostrado el conocimiento e interés de los poderes públicos españoles de la época por las acciones contables de los mercaderes, hasta el punto de dictar con una antelación de más de doscientos años sobre sus más inmediatos seguidores la primera legislación en el mundo imponiendo a los comerciantes la obligación de llevar libros de cuentas - contables no conocidos como tal- y de hacerlo precisamente por el método de partida doble (pragmáticas de Cigales de 1549 y de Madrid de 1552). Con esta legislación, que fija un hito y supone uno de los grandes momentos estelares de la historia de la contabilidad española en el contexto mundial, se pretendía impedir o, al menos, aminorar la salida fraudulenta de metales preciosos más allá de las fronteras, habida cuenta del rastro indeleble que la contabilidad por partida doble dejaba de todas las operaciones.

El estudio de la historia de la contabilidad proporciona, asimismo, una visión desconocida e inesperada del rey Felipe II, que, con la imagen enfática y distante que la historia le ha asignado tradicionalmente, era capaz, sin embargo, de estar perfectamente al corriente de las prácticas mercantiles de su tiempo y de interesarse profundamente por ellas. A él se le debe la anexión a la administración pública de relevantes hombres de negocios para aprovechar sus 


\section{La Contabilidad Internacional: incluye visión histórica, paradigma y teorías}

Vol. 3, núm. 3., (2019)

Clara de las Mercedes Razo Ascazubi; Jeanette Lorena Moscoso Córdova; Patricia Geraldina López Fraga

conocimientos, así como la introducción en 1592, tras un intento fallido en 1580, de la contabilidad por partida doble para llevar las cuentas centrales de su Real Hacienda, como primer gran país en el mundo, en lo que constituye otro de los momentos estelares de la historia de la contabilidad en España. Estos hechos dan idea del grado de interés y atención con que en la Corte española se seguían las novedades en el mundo mercantil y financiero.

La decadencia generalizada del comercio ultramarino de Cádiz en las primeras décadas del siglo XIX tras la pérdida de las colonias americanas, ocasionando el rápido declive de la ciudad que perdió su status de gran metrópoli próspera y opulenta, ha constituido desde siempre uno de los tópicos de nuestra historia económica contemporánea. Sin embargo, el reciente estudio de la documentación contable de una empresa comercial gaditana establecida en el año 1730 y que se ha mantenido viva hasta nuestros días ha puesto de manifiesto que sus años de mayor prosperidad y actividad con Hispanoamérica fueron precisamente los de la segunda mitad del siglo XIX. No debió de ser, pues, tan fatal y absoluta la decadencia mercantil de Cádiz como consecuencia de la emancipación colonial americana. Este es otro ejemplo de cómo la investigación de las fuentes contables ha venido a corregir y arrojar sombras sobre las percepciones históricas establecidas, que habrá que revisar.

Para finalizar este breve repaso de ejemplos en los que la investigación histórico-contable ha venido a completar o, incluso, a cambiar el signo de las interpretaciones históricas recibidas, un caso en el que los estudios de historiadores españoles de la contabilidad han contribuido a desacreditar una teoría ampliamente extendida entre los historiadores de la empresa norteamericanos: la de que las técnicas avanzadas de gestión empresarial, y entre ellas la contabilidad de costes, habían surgido en el siglo XIX como consecuencia de la presión ejercida 


\section{La Contabilidad Internacional: incluye visión histórica, paradigma y teorías}

Vol. 3, núm. 3., (2019)

Clara de las Mercedes Razo Ascazubi; Jeanette Lorena Moscoso Córdova; Patricia Geraldina López Fraga

por la libre competencia entre las grandes empresas. Los archivos contables de la Real Fábrica de Tabacos de Sevilla han venido a demostrar cómo, en los siglos XVII y XVIII, una gran empresa en régimen de monopolio estatal practicaba ya un riguroso control de costes y unas desarrolladas técnicas de gestión.

Ahora bien, ¿por qué si las ventajas de incorporar al análisis histórico-económico el estudio e interpretación de la documentación contable parecen tan evidentes y proporcionan resultados tan gratificantes, la historia de la contabilidad, pese al auge experimentado en los últimos años, es relativamente tan poco conocida y practicada todavía...?

La respuesta debe buscarse, tal vez, en las dificultades técnicas que la contabilidad ofrece al no iniciado, dificultades que se multiplican cuando lo que hay que interpretar requiere el conocimiento de las técnicas vigentes en determinados períodos del pasado. Esta dificultad ha dado lugar, precisamente, a que algunos hayan dado a la historia de la contabilidad el apelativo de ingeniería de la historia económica, por considerarla su parte más difícil, intrincada y recóndita, como efectivamente así es. Tales dificultades técnicas son las que han motivado que muchos historiadores económicos hayan soslayado y sigan eludiendo en sus investigaciones los aspectos histórico-contables para no introducirse en un terreno resbaladizo que no conocen bien.

\section{La llegada de la partida doble}

Las anotaciones contables se diferenciaron desde el principio al objeto de adaptarse a dos finalidades fundamentales: 


\section{La Contabilidad Internacional: incluye visión histórica, paradigma y teorías}

Vol. 3, núm. 3., (2019)

Clara de las Mercedes Razo Ascazubi; Jeanette Lorena Moscoso Córdova; Patricia Geraldina López Fraga

a) Satisfacer la obligación de agentes y factores de rendir cuentas a sus principales acerca de la gestión de los bienes y haciendas confiados a su cuidado, y,

b) Seguir de cerca la marcha global de los propios negocios o actividades.

Aunque con numerosos puntos de contacto y solapamiento, ambas finalidades dieron lugar a dos distintos sistemas de registro contable, con características propias y específicas.

Común a ambas clases de registros, en principio, el perfil bilateral de las anotaciones, que consideraban simplemente la relación entre dos personas, el dueño de los libros y un tercero. Pero mientras el primer sistema se mantuvo en este planteamiento bilateral, el segundo fue evolucionando en busca de un grado cada vez mayor de integración, de multilateralidad, hasta que las anotaciones abarcaron todas las operaciones del dueño de los libros, ofreciendo una visión completa e interrelacionada de sus negocios.

No se crea por ello, sin embargo, que el primer sistema no evolucionó. Lo hizo y se fue perfeccionando, pero conservando siempre su carácter bilateral, que le restaba utilidad para su empleo como método de registro, memoria y gestión empresariales. Por ello, su utilización se desarrolló sobre todo al servicio de las administraciones públicas.

En tanto, su planteamiento se ajustaba perfectamente a la necesidad de controlar la acción de los empleados públicos y de las personas a las que se encomendaba una misión que involucrase la recepción, entrega y manejo de los dineros o bienes públicos. En los reinos de Castilla, estas acciones contables originaron, ya avanzada la Edad Media, el llamado sistema de 


\section{La Contabilidad Internacional: incluye visión histórica, paradigma y teorías}

Vol. 3, núm. 3., (2019)

Clara de las Mercedes Razo Ascazubi; Jeanette Lorena Moscoso Córdova; Patricia Geraldina

López Fraga

Cargo y Data, que se ha mantenido en la Administración de una forma u otra hasta bien entrado el siglo $\mathrm{XX}$.

Con relación a la segunda visión de los registros contables, es decir, los destinados a facilitar una visión de la marcha de los negocios y actividades, mantuvieron en lo esencial un carácter bilateral posiblemente hasta los siglos XI o XII.

Desde esta época se introduce, lentamente, elementos integradores. La integración completa no se consigue, sin embargo, hasta finales del siglo XIII o comienzos del XIV con el nacimiento de la contabilidad por partida doble. Este sistema contable -aplicado aun en la actualidad con elementos actualizados, incluso por avances de las tecnologías de información y comunicación -, integra e interrelaciona todos los elementos que constituyen el acervo patrimonial, la caja, la mercaderías, los deudores, los acreedores, entre otros, registrando tanto los cambios de estructura del patrimonio, es decir, aumentos, por ejemplo, de Mercaderías con una correlativa disminución de la Caja debido a una compra en efectivo de aquéllas, como las variaciones en el volumen total del patrimonio, representado por la cuenta de Capital.

Esta fue, precisamente, la gran innovación aportada por la partida doble, pues las visiones anteriores se encargaban solo de registrar cambios de estructura del patrimonio. Desde éste perspectiva la situación podría parecer obvio y elemental, y tal vez lo sea, pero lo cierto es que lo último del nuevo planteamiento requirió siglos de esfuerzos y tanteos, constituyendo una auténtica revolución conceptual, un cambio de filosofía contable.

El carácter completo y que incluye todo en la contabilidad por partida doble hizo que cobraran sentido medidas adicionales para garantizar la fiabilidad de los libros. Así, se 


\section{La Contabilidad Internacional: incluye visión histórica, paradigma y teorías}

Vol. 3, núm. 3., (2019)

Clara de las Mercedes Razo Ascazubi; Jeanette Lorena Moscoso Córdova; Patricia Geraldina López Fraga

constituyó en norma consuetudinaria y aun legal, en algunos casos, el hecho de que los libros diario y mayor, libros típicos que se hicieron con el tiempo obligatorios y principales de la partida doble, estuvieran encuadernados, sin tachaduras, sin dejar hojas ni espacios en blanco, entre otros, todo ello, para no incorporar hojas nuevas ni sustituir las originales; tampoco que existiera la posibilidad de anular partidas ya registradas o incorporar asientos nuevos. Asimismo, se generalizó la acción de legitimar los libros en los Consulados u otras organizaciones de mercaderes. De este modo, los libros de cuentas -hoy de contabilidad, de uso obligatorio y al día- se convirtieron en elementos probatorios ante los tribunales de la jurisdicción respectiva.

La epistemología y metodología contable: entre paradigmas y teorías de las ciencias contables

En los dos últimos decenios del siglo XX, el aspecto tradicional de la contabilidad se ha visto sacudido por una revuelto en el seno de la disciplina contable, con el fraccionamiento en dos historias, que ha provocado efectos estimulantes sobre el interés despertado por el estudio de la disciplina.

En consecuencia, hacemos referencia a la aparición de la "nueva historia de la contabilidad", que surge con fuerza y como un movimiento retador con la historia de la contabilidad tradicional, abriendo un agrio debate epistemológico y metodológico sin precedentes.

La "nueva historia de la contabilidad" se constituye de la corriente postmoderna del relativismo y contestación a los cánones y planteamientos culturales heredados; adicionalmente, movimientos que durante las últimas décadas han invadido todos los ámbitos intelectuales y hasta artísticos. 


\section{La Contabilidad Internacional: incluye visión histórica, paradigma y teorías}

Vol. 3, núm. 3., (2019)

Clara de las Mercedes Razo Ascazubi; Jeanette Lorena Moscoso Córdova; Patricia Geraldina López Fraga

Dentro de su medio específico, la "nueva historia de la contabilidad" ha nacido del regimiento y conexión de las nuevas ideas epistemológicas y metodológicas sobre la historia en general con el nuevo paradigma crítico interpretativo de investigación de la contabilidad, de clara orientación marxista.

Las posturas filosóficas se han constituido a los fines de compartir y discutir distintos razonamientos lógicos y métodos sobre nociones abstractas directamente correspondidos con la humanidad y el entorno que nos rodea. A continuación se muestra la Figura 1, la cual expresan algunas posiciones filosóficas de la ciencia con incidencia en el campo contable.

Figura 1. Corrientes filosóficas

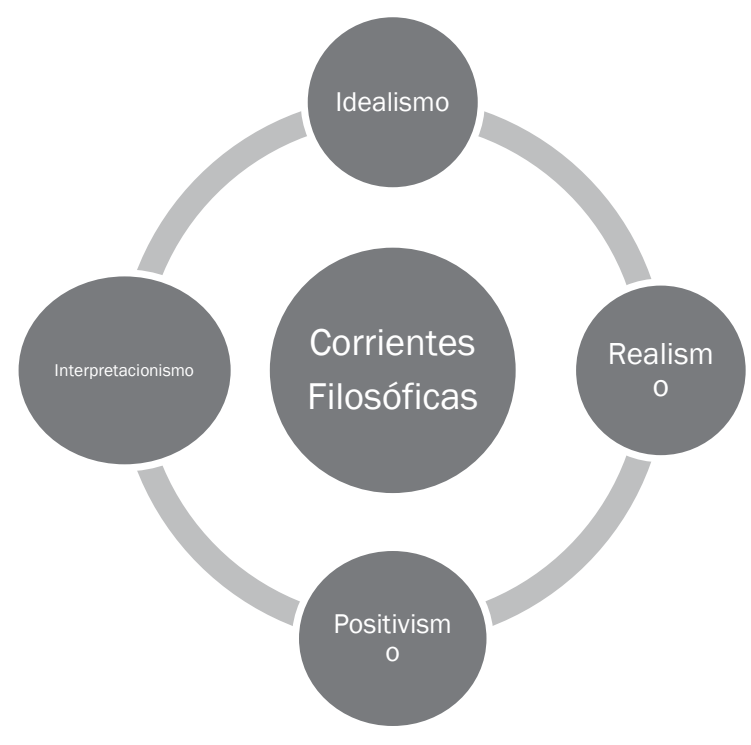

Fuente: elaboración propia

En este orden de ideas, en el idealismo los pensamientos representan el punto de partida y el principio de toda reflexión sobre de la realidad. Se fundamenta en que la realidad es mental, o 


\section{La Contabilidad Internacional: incluye visión histórica, paradigma y teorías}

Vol. 3, núm. 3., (2019)

Clara de las Mercedes Razo Ascazubi; Jeanette Lorena Moscoso Córdova; Patricia Geraldina López Fraga

que se enuncia y concibe mejor como una idea, es decir, que la verdadera realidad se encuentra en el concepto que de ella se tiene.

Con relación al realismo, el mismo muestra la manera de narrar, exhibir, razonar u observar lo que ocurre tal como sucede, sin ningún idealismo. A partir de acá se puede indicar que la corriente realista posee la característica de soslayar exageraciones: sólo relata los sucesos concretos.

Cabe destacar que el realismo también se reconoce por destacar la existencia objetiva de los conceptos de carácter universal. Desde la óptica de la filosofía moderna, el realismo establece un saber fundamentado en la idea de que los objetos que pueden observarse por medio de los sentidos tienen una presencia que resulta independiente con relación a ellos mismos.

En cuanto al positivismo, ésta corriente filosófica parte desde la perspectiva que el conocimiento científico se deriva de los acontecimientos descubiertos por la observación y la experimentación buscando la verdad mediante la racionalidad científica, con sus abordajes y perspectivas particulares, como por ejemplo el uso del método científico -con carácter únicopara todas las ciencias. Es la corriente de pensamiento que ha perdurado en el tiempo transmitiéndose de teoría en teoría usando el empirismo como método. Ésta corriente ha incidido en la práctica contable al revelar los hechos a través de leyes y principios. De aquí, se reconoce a las bases contables que dan soporte, carácter y expansión a la ciencia contable desde los Principios de Contabilidad Generalmente Aceptados (PCGA).

En contraposición al positivismo, frente a esta corriente surgen otros enfoques que establecen el estudio, comprensión e interpretación de los elementos considerados para dejar 


\section{La Contabilidad Internacional: incluye visión histórica, paradigma y teorías}

Vol. 3, núm. 3., (2019)

Clara de las Mercedes Razo Ascazubi; Jeanette Lorena Moscoso Córdova; Patricia Geraldina López Fraga

tradicionalmente el aspecto meramente contable representado en números sin sentido que provocaron un escaso reconocimiento de la contabilidad como ciencia. En tal sentido, el pensamiento interpretacionista incorpora el elemento cualitativo superando el método contable netamente cuantitativo.

Éste enfoque del conocimiento se basa en la interpretación detallada de la realidad y de las causas que la han llevado a ser así, sin una visión general y de explicaciones deterministascausales-lineales, es decir, los estudios que se ejecutan son de carácter interdisciplinarios y transdisciplinarios, donde predomina la existencia de investigadores que siguen el paradigma formando parte de los objetos que se observan a través de la interacción.

Partiendo del pensamiento Kuhniano del desarrollo científico, un paradigma puede definirse como un patrón, modelo de referencia o conjunto de reglas y procedimientos que son aplicados para la explicación de la realidad de un fenómeno o para la solución de un problema (Kuhn T., 1962). En la ciencia contable, son diversos los paradigmas que han influido en su evolución, lo que ha permito en el tiempo asentarse como tal.

En la ciencia contable existen algunos investigadores que de acuerdo con su juicio proponen a su manera enfoques doctrinales para clasificar sus desarrollos teóricos; podemos señalar entre otros: Richard Mattessich con "Tradiciones de investigación". Leandro Cañibano con sus "Programas de investigación contable", Vicente Montesinos Julve con "Corrientes doctrinales" y Ahmed Belkaoui con "Paradigmas contables"; todas contribuyeron de alguna manera para el reconocimiento de la contabilidad como ciencia y no como una práctica artística de los números. 


\section{La Contabilidad Internacional: incluye visión histórica, paradigma y teorías}

Vol. 3, núm. 3., (2019)

Clara de las Mercedes Razo Ascazubi; Jeanette Lorena Moscoso Córdova; Patricia Geraldina López Fraga

Partiendo de lo anterior, los dos grandes paradigmas, a nuestra consideración, esbozados en ésta investigación se muestran seguidamente en la figura 2.

Figura 2. Paradigmas contables y sus enfoques

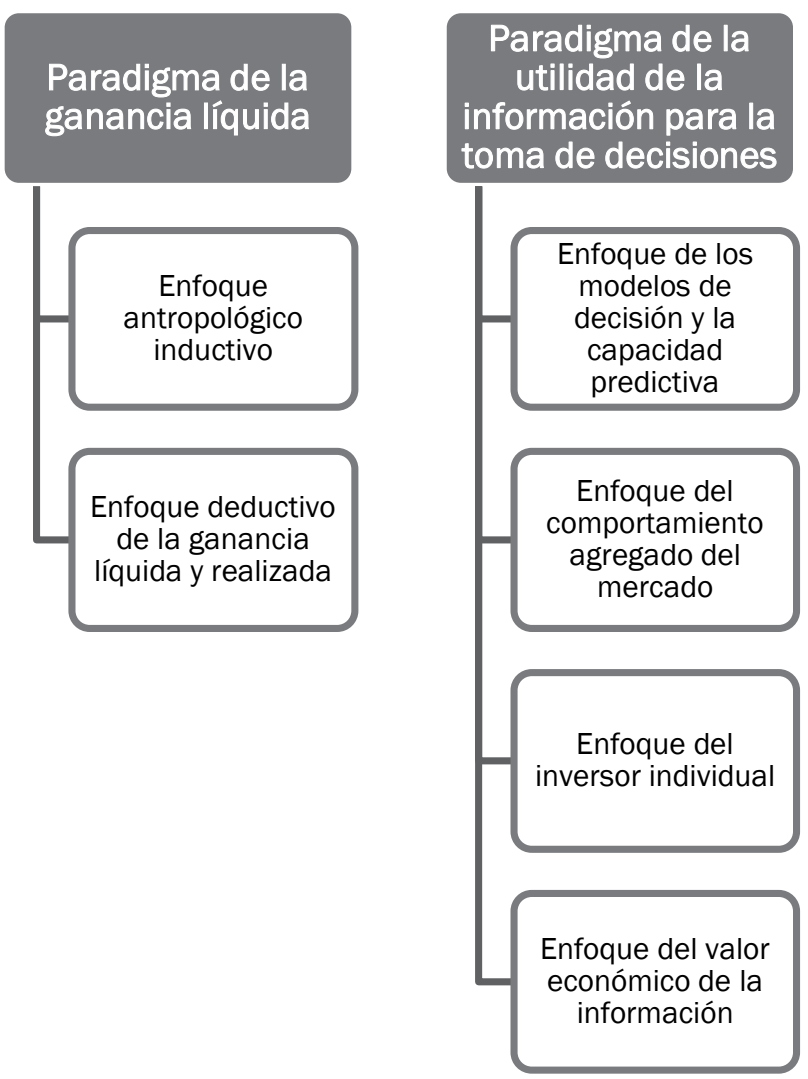

Fuente: elaboración propia adaptado de Santos y otros (1997)

\section{a. Paradigma de la ganancia líquida}

De acuerdo con Santos y otros (1997), esta corriente del pensamiento contable tuvo como objetivo la producción de información contable con relación al beneficio económico producido por una entidad, concebido este último desde una visión exclusivamente jurídica. (p. 142) 


\section{La Contabilidad Internacional: incluye visión histórica, paradigma y teorías}

Vol. 3, núm. 3., (2019)

Clara de las Mercedes Razo Ascazubi; Jeanette Lorena Moscoso Córdova; Patricia Geraldina

López Fraga

La escuela predominante de éste paradigma corresponde a la Escuela JurídicoPersonalista, la cual fue postulada por Giuseppe Cerboni, en el que se propone que la información generada desde la contabilidad partiendo de los resultados obtenidos en un periodo pueda utilizarse como medio probatorio en los distintos escenarios.

Otra vertiente predominante bajo el paradigma de la ganancia líquida, está centrada en la Escuela Contista, en la cual concibe a la contabilidad como la ciencia de las cuentas, de los números, con la firme convicción de la elaboración y aplicación de normas que regulen los registros contables.

En ésta misma dirección, encontramos dentro de este paradigma dos grandes enfoques identificados como:

a.1. Enfoque antropológico inductivo, y,

a.2. Enfoque deductivo de la ganancia líquida y realizada.

Según señala Belkaoui, el estudio base del enfoque antropológico inductivo se fundamenta en la presencia de prácticas contables y de acciones gerenciales hacia esas prácticas. Dicho enfoque busca comprender, revelar y estimar las prácticas contables existentes. Se pueden identificar dos clases de teorías dentro de este enfoque:

- La que pretende explicar y justificar las prácticas contables en uso, como por ejemplo: la valoración de activos, el costo histórico, técnicas en el asiento de libros, entre otros. Aquí se emplea técnicas analíticas, métodos observacionales e investigación por encuestas para el estudio del objeto de estudio. 


\section{La Contabilidad Internacional: incluye visión histórica, paradigma y teorías}

Vol. 3, núm. 3., (2019)

Clara de las Mercedes Razo Ascazubi; Jeanette Lorena Moscoso Córdova; Patricia Geraldina López Fraga

- La que pretende explicar cómo influye la gerencia en la generación de técnicas, considerando la suposición del beneficio equilibrado y los inicios de una teoría positiva de la contabilidad desde técnicas empíricas.

Por otro lado, con respecto al enfoque deductivo de la ganancia líquida y realizada, su objeto de estudio se basa en un concepto ideal de renta fundamentado en otro distinto al costo histórico. Belkaoui muestra cinco postulados bajo este enfoque:

- $\quad$ Contabilidad ajustada a nivel de precios

- $\quad$ Contabilidad de costos de remplazo

- $\quad$ Contabilidad de valores de salida

- $\quad$ Contabilidad del valor neto realizable, $y$,

- $\quad$ Contabilidad del valor presente.

Cada una de estas teorías presenta distintas formas de valoración de activos y determinación de la renta arguyendo a su vez la superación de los defectos del sistema del costo histórico. La metodología empleada bajo ésta óptica es el razonamiento analítico, recurriendo a los objetivos y premisas del entorno concluyendo con métodos específicos. Este paradigma pone de manifiesto el registro contable donde el beneficio económico es el preponderante de la información, de tal forma, la contabilidad muestra un carácter orientado a la descripción.

\section{b. Paradigma de la utilidad de la información para la toma de decisiones}




\section{La Contabilidad Internacional: incluye visión histórica, paradigma y teorías}

Vol. 3, núm. 3., (2019)

Clara de las Mercedes Razo Ascazubi; Jeanette Lorena Moscoso Córdova; Patricia Geraldina López Fraga

Su primordial objetivo es proveer información desde el punto de vista cuantitativo, que sea provechosa para la toma de decisiones. Bajo este esquema de pensamiento, muestra la contabilidad como un agregado interdisciplinario, puesto que demanda la participación de otras disciplinas como las matemáticas, álgebra, estadística, econometría, investigación operativa, sociología, psicología, ciencias políticas y la comunicación.

Al respecto, del paradigma para la toma de decisiones se reconocen 4 perspectivas para comprender el mismo, de los cuales tenemos:

\section{b.1. Enfoque de los modelos de decisión y la capacidad predictiva}

Su objetivo es hacer estudios de estimación de la información contable como instrumento básico para la toma de decisiones, es decir, empleando la estadística, fija un valor de confiabilidad mucho más alto a la información contable que tenga la capacidad de predecir los acontecimientos futuros. Considerando los argumentos expuestos, las guías de decisión examinan la información a los fines de evaluar la posible utilidad y relevancia que conceda un carácter predictivo a la misma.

\section{b.2. Enfoque del comportamiento agregado del mercado}

Desde esta postura, se estudia la incidencia de la información contable en los precios de los títulos valores comercializados en el campo bursátil; es decir, se analiza los hechos financieros del mercado bursátil como parte fundamental de la economía.

\section{b.3. Enfoque del inversor individual}




\section{La Contabilidad Internacional: incluye visión histórica, paradigma y teorías}

Vol. 3, núm. 3., (2019)

Clara de las Mercedes Razo Ascazubi; Jeanette Lorena Moscoso Córdova; Patricia Geraldina López Fraga

Entre las principales características que presenta esta vertiente, busca estudiar los efectos que sobre el usuario -visto individualmente-, origina la información contable, así como las causas productoras de la misma. En este enfoque se conocen dos teorías principales:

a) La teoría del comportamiento, y,

b) La teoría de la información.

En tales teorías identifica el conocimiento y la comprensión de la naturaleza del hombre, representadas en motivaciones, necesidades, estímulos, prohibiciones, el contexto cultural, entre otros. En tal sentido, Santos y otros (1997), indican que estas teorías respaldan el poder de decisión de los individuos, los cuales deben ser considerados por la contabilidad, que recibe la información originada por las relaciones y condiciones sociales. (p. 145). Ejemplificando, la contabilidad procesa información y comunica al individuo en calidad de usuario para responder a sus necesidades y motivaciones. En atención a lo anterior, es de considerar la existencia de limitaciones contables donde el usuario de la información deberá soslayar para que su decisión tenga la capacidad de influir de forma más atinada sobre el medio.

\section{b.4. $\quad$ Enfoque del valor económico de la información}

Las características expuestas sobre este enfoque, son consideradas ampliamente por Tua Pereda (1988) de acuerdo con la siguiente caracterización:

- La información es vista como un bien económico escaso, con un costo y como contrapeso ofrece un beneficio a los usuarios para la toma de decisiones. 


\section{La Contabilidad Internacional: incluye visión histórica, paradigma y teorías}

Vol. 3, núm. 3., (2019)

Clara de las Mercedes Razo Ascazubi; Jeanette Lorena Moscoso Córdova; Patricia Geraldina López Fraga

- Desde la racionalidad del inversionista, se obtiene que la generación de información está normada por el juego de la oferta y demanda establecidas por los consumidores, los cuales pretenden maximizar sus funciones de utilidad, de tal forma que toda acción debe exceder el valor de su costo.

- La información contable se analiza en su carácter para acrecentar la propiedad de lograr elecciones óptimas, siendo necesarias para el estudio sistemático de la probabilidad la posibilidad de elegir entre un abanico de opciones.

- El usuario escoge entre distintas acciones con diferentes resultados posibles, obteniendo una conducta racional del mismo, persiguiendo el mayor beneficio. En este contexto, la información es primordial, al punto de analizar las probabilidades de cada uno de los beneficios deseados.

- $\quad$ En efecto, el usuario hace frente a dos fases, una en la que el sistema de información permite observar distintas señas, y la otra, en la que la observación de los resultados de una señal determinada conlleva al estudio de las probabilidades y a la elección de una acción dependientemente óptima.

- Con estas apreciaciones, el enfoque de la economía de la información puede trazar, bien sea en el análisis de cada sistema de información específico, para conseguir su valor, o bien el sistema de información óptimo entre varias posibilidades. 


\section{La Contabilidad Internacional: incluye visión histórica, paradigma y teorías}

Vol. 3, núm. 3., (2019)

Clara de las Mercedes Razo Ascazubi; Jeanette Lorena Moscoso Córdova; Patricia Geraldina López Fraga

- $\quad$ El sistema óptimo será aquel con mayor diferencia entre el costo de la información y el beneficio generado por la ella, en términos de maximización de utilidad esperada.

- $\quad$ Con lo expuesto, se soluciona la interrogante planteada por el paradigma de la utilidad, formando de este modo cuál puede ser el origen de la información más apropiada al inversionista, partiendo de un planteamiento fundamentado en el análisis de costo-beneficio.

En conclusión, ante la caracterización hecha anteriormente, podemos señalar que la información contable asume particularidades de mercancía, es decir, puede escasear, poseer costo, ser útil, notable, ineludible, codiciada y óptima; y con la cual la persona pretende maximizar su utilidad y obtener mejores beneficios.

Una vez analizadas las principales corriente filosóficas y los paradigmas contables, nos adentramos a otros elementos que fortalecen el andamiaje epistemológico contable relacionado con las teorías contables.

En este orden de ideas, cabe señalar que diversos estudios fueron dirigidos sobre todo a considerar los aspectos económicos y administrativos, pero desde una óptica normativa-contable dirigida al cómo hacer las cosas. Tal situación, produjo consecuencias sobre la visión científica de la contabilidad, en este contexto, los estudiosos del tema han propuesto la teoría crítica y la crítica interpretativa como teorías emergentes y alternativas para dar respuesta a la práctica profesional. 


\section{La Contabilidad Internacional: incluye visión histórica, paradigma y teorías}

Vol. 3, núm. 3., (2019)

Clara de las Mercedes Razo Ascazubi; Jeanette Lorena Moscoso Córdova; Patricia Geraldina López Fraga

En la teoría interpretativa prevalece la generación de conocimiento desde lo subjetivo, procurando efectuar estudios que pueda describirse, comprenderse e interpretarse el significado de los sujetos en el entorno considerando como base fundamental las experiencias vividas por los sujetos de estudio.

En esta misma dirección, la corriente crítico-interpretativa busca rescatar las experiencias en la que Habermas (1987) a partir de la acción comunicativa emplea la lingüística para la interpretación, eso sí, enlazada con pautas, valores, entes y experiencias de nociones o conceptos acompañados por todos.

Finalmente, en la vertiente crítico-interpretativa, el conocimiento que se genera está estrechamente afectado por las relaciones de poder, por consiguiente, los acontecimientos no pueden ser apartados de los valores y de la ideología que influye en los grupos sociales.

Como circunstancia curiosa, debe señalarse que los fundadores de la nueva historia tienen a gala proclamar que su grupo no está compuesto por historiadores, sino por sociólogos, politólogos, etnólogos, ideólogos, etc., queriendo, posiblemente, hacer hincapié con ello en el carácter multidisciplinar de sus investigaciones.

La "nueva historia de la contabilidad" no constituye, en realidad, una escuela o un cuerpo unitario de doctrina, sino un conjunto variado de enfoques y formas de entender la disciplina que, a menudo, muestran importantes diferencias entre sí. Pero, en cualquier caso, lo cierto es que sus partidarios comparten unas ideas y tendencias comunes que hacen que este conjunto de enfoques y planteamientos se distinga de las formas tradicionales en que la historia de la contabilidad se había venido entendiendo y practicando. 


\section{La Contabilidad Internacional: incluye visión histórica, paradigma y teorías}

Vol. 3, núm. 3., (2019)

Clara de las Mercedes Razo Ascazubi; Jeanette Lorena Moscoso Córdova; Patricia Geraldina López Fraga

Los dos grandes rasgos distintivos de la "nueva historia de la contabilidad" en relación con la "tradicional" son posiblemente los siguientes:

a) La pérdida de la fe en la posibilidad de alcanzar la objetividad en el estudio y descripción de los hechos históricos.

b) El abandono de la idea de que la contabilidad, lo mismo que las demás actividades humanas, está sujeta a un proceso más o menos lineal de progreso continuado bajo el estímulo de las necesidades de adaptación a los requerimientos del entorno.

Los adeptos de la "nueva historia de la contabilidad" niegan la existencia de este proceso evolutivo hacia el progreso y por ello postulan que lo significativo, lo que debe estudiarse, no son los cambios evolutivos, sino las situaciones de ruptura, de interrupción, de retroceso. Por otra parte, no creen tan importante la adaptación de la contabilidad a los requerimientos del mundo exterior, como la forma en que la contabilidad ha contribuido y sigue contribuyendo a influenciar su entorno y, por ende, la configuración y evolución de la sociedad.

En la adopción de estos planteamientos anti-evolutivos han tenido especial importancia las ideas de Michel Foucault, que, de una manera u otra, han influido en casi todos los adeptos a la "nueva historia de la contabilidad", ejerciendo en algunos de ellos un influjo tan fuerte que los ha configurado como un grupo específico dentro del conjunto. En el contexto que nos ocupa, las ideas de Foucault que más han afectado a los nuevos historiadores de la contabilidad en general han sido las relativas a las relaciones saber-poder y al papel de la disciplina en la sociedad de nuestros días. En efecto, desde que la humanidad hiciera estallar a finales del siglo XVIII el 


\section{La Contabilidad Internacional: incluye visión histórica, paradigma y teorías}

Vol. 3, núm. 3., (2019)

Clara de las Mercedes Razo Ascazubi; Jeanette Lorena Moscoso Córdova; Patricia Geraldina López Fraga

corsé de los gobiernos autoritarios para hacer valer los ideales de libertad, las capas dirigentes de la sociedad han tenido que ir arbitrando nuevas formas indirectas de disciplinar y gobernar a sus miembros. La contabilidad forma parte de este proceso, que Foucault ilustra a partir del Panopticon de Jeremy Bentham, es decir, de esa prisión circular en la que los guardianes pueden ver y vigilar a los presos sin ser vistos, de forma que éstos se sientan constantemente vigilados.

La contabilidad directiva o de gestión ofrece a los foucaultianos un ejemplo patente de este proceso dentro del contexto contable, pues dicho sistema permite observar el funcionamiento de toda la organización y asegurar la vigilancia y el control del comportamiento y rendimiento de sus trabajadores. Naturalmente, para los foucaultianos, la contabilidad de gestión no ha sido desarrollada por motivos de racionalidad puramente económica, sino más bien como consecuencia de esa búsqueda, nunca explicitada por las capas dirigentes, de medios de disciplina, control y vigilancia sociales.

Por otra parte, el derrumbamiento y rechazo de la llamada "ilusión de la objetividad" "that noble dream", como la califica burlonamente Peter Novick- han hecho restar importancia a la investigación y exposición de los hechos histórico-contables y han cambiado la actitud del nuevo historiador hacia los mismos. No se trata ya de "dejar hablar a los hechos por sí mismos", sino de formular interpretaciones apriorísticas que puedan ser verificadas por los hechos. La reflexión teórica debe preceder, así, siempre a la búsqueda y recopilación de los hechos. Esta posición es la que hace que los nuevos historiadores de la contabilidad desprecien la investigación en archivos y la búsqueda de fuentes primarias, que tachan de inútil y anticuaria, mero anhelo de acumular erudición vacía y sin sentido, proclamando que la única actividad histórica con verdadera significación es la interpretación de los hechos. Aunque, acto seguido, 


\section{La Contabilidad Internacional: incluye visión histórica, paradigma y teorías}

Vol. 3, núm. 3., (2019)

Clara de las Mercedes Razo Ascazubi; Jeanette Lorena Moscoso Córdova; Patricia Geraldina López Fraga

ponen de manifiesto claramente sus verdaderos intereses, pues, en su afán de socavar los cimientos culturales del mundo occidental al objeto de edificar una sociedad más justa, más solidaria y más igualitaria, no se esfuerzan en ocultar que la única interpretación de los hechos que les interesa es la que parte de los planteamientos marxistas de explotación y lucha de clases.

Es difícil prever lo que traerá el futuro, pero las modas pasan y las pugnas ceden. Sólo lo auténtico permanece. Esto es lo que ocurrirá, posiblemente, con esta pugna en el seno de la historia de la contabilidad, cuando las ideas postmodernistas hayan demostrado su inconsistencia y se haya comprendido que negar la posibilidad de lograr una dosis suficiente de objetividad en la investigación histórica supone la negación misma del conocimiento histórico. Del debate actual quedará simplemente el impulso dado al estudio y a la divulgación de la historia de la contabilidad.

\section{La contabilidad internacional}

Bajo éste tipo de contabilidad se busca proyectar el pensamiento local, pero con una visión globalizada desde las corrientes filosóficas que hasta el momento han sido estudiadas. Para ello, a través de los fundamentos epistemológicos expresados, se busca argumentar la postura internacional desde la doctrina y teorías que respaldan el ejercicio profesional en un contexto globalizado e integral en el ámbito internacional.

En la medida en que la internacionalización empresarial se ha ido intensificando y va surgiendo un nuevo usuario de la información internacional, es importante situar a las empresas en el escenario que la nueva dimensión internacional de la información contable está creando y necesitando. Uno de los principales objetivos de la contabilidad, es proporcionar información útil 


\section{La Contabilidad Internacional: incluye visión histórica, paradigma y teorías}

Vol. 3, núm. 3., (2019)

Clara de las Mercedes Razo Ascazubi; Jeanette Lorena Moscoso Córdova; Patricia Geraldina López Fraga

para la toma de decisiones por parte de los usuarios. Esto adquiere mayor relevancia en la utilización de la información contable aplicada a las Normas Internacionales, la cual no se ciñe sólo al ámbito nacional, sino que va más allá de las fronteras; la evolución del entorno que rodea a la empresa ha propiciado en los últimos años un proceso de internacionalización de la actividad económica, como consecuencia de la información contable útil.

Para Tua y Gonzalo (1988) reseñan la definición de Choi-Mueller (1984) en la cual explica que el término de Contabilidad internacional se extiende, en su más amplio sentido, hasta abarcar la contabilidad para propósitos generales y orientados a términos nacionales, dirigida a:

a) El análisis comparativo internacional

b) Desarrollar temas de medida e información contable relativos exclusivamente a las transacciones de empresas multinacionales y a la forma de los negocios de dichas empresas

c) A cubrir las necesidades contables de los mercados financieros internacionales

d) A la armonización de la diversidad en cuanto a contabilidad e información financiera, a conseguir mediante acciones políticas, organizativas, profesionales y de fijación de normas.

e) Reconociendo la importancia de la contabilidad internacional, Tua y Gonzalo presentan sucintamente las áreas posibles de este campo: 


\section{La Contabilidad Internacional: incluye visión histórica, paradigma y teorías}

Vol. 3, núm. 3., (2019)

Clara de las Mercedes Razo Ascazubi; Jeanette Lorena Moscoso Córdova; Patricia Geraldina López Fraga

a. Sistemas contables: incluye aspectos históricos de clasificación, análisis, comparación y predicción acerca de la evolución del conjunto de prácticas contables seguidas en cada país o grupo de países considerados como sistemas contables.

b. Teoría e instituciones de la normalización contable internacional en el ámbito externo: área que aborda la historia, cometido, metodología, tareas e influencias ejercidas por los organismos que confeccionan normas de contabilidad financiera con validez simultánea en varios países.

En los servicios contables, con la aplicación de la teoría de la globalización, es necesario darle cabida a la contabilidad internacional con su labor en las organizaciones internacionales.

Finalmente, lo que termina dándole un sentido a la contabilidad como internacional, se corresponde con la aparición y la transición de prácticas contables a las que deben someterse a través de las Normas Internacionales de Información Financiera (NIIF).

\section{Conclusiones.}

A lo largo del desarrollo de la investigación, se ha podido evidenciar la existencia de cuatro grandes perspectivas filosociológicas, que han puesto de manifiesto el desarrollo de la disciplina contable en su historia, y mostrando la importancia de la contabilidad internacional en el trascurrir del tiempo. 


\section{La Contabilidad Internacional: incluye visión histórica, paradigma y teorías}

Vol. 3, núm. 3., (2019)

Clara de las Mercedes Razo Ascazubi; Jeanette Lorena Moscoso Córdova; Patricia Geraldina López Fraga

Una distinción fundamental entre lo natural y los fenómenos sociales es que un entendimiento de los fenómenos sociales requiere una apreciación de los significados atribuidos a esos fenómenos por individuos afectados por ellos además de un entendimiento de los afloramientos observables de esos fenómenos. Los valores connotados por lo social las acciones son un aspecto crucial del significado de esas acciones $\mathrm{y}$, por tanto, el comportamiento de los individuos y la respuesta afectiva a las acciones, las corrientes esbozadas anteriormente son intentos de conceptualizar los medios por los cuales los valores o intereses se incorporan en el conocimiento y promulgado en las prácticas sociales del día a día.

Cada una de estas perspectivas ha sido utilizada para examinar el papel de la contabilidad internacional en el campo de las organizaciones. Las metodologías de las ciencias naturales pueden no ser apropiadas para cuestiones de significado y valores. En muchos casos, además, los valores que abarca la contabilidad y los actos son implícitos en la acción y, por lo tanto, invisible a las metodologías convencionales como una forma de solucionar este problema puede ser enfoque en "situaciones críticas" (Giddens, 1979) donde se interrumpen las rutinas sociales y lo implícito se vuelve problemático.

De estas perspectivas, en ningún caso tienen sus ideas que hayan sido explotados sistemáticamente. El desarrollo de una sociología de la contabilidad dentro de cada una de estas tradiciones contribuiría significativamente a nuestra comprensión de la contabilidad como un proceso social y organizativo. 


\section{La Contabilidad Internacional: incluye visión histórica, paradigma y teorías}

Vol. 3, núm. 3., (2019)

Clara de las Mercedes Razo Ascazubi; Jeanette Lorena Moscoso Córdova; Patricia Geraldina López Fraga

\section{Bibliografía.}

Argyris, C. (1976), Single Loop and Double Loop Models in Research on Decision-Maldng, Administrative Science Quarterly pp. 363-375.

Berger, P. \& Luckmann, T. (1966)., The Social Construction of Reality (New York: Penguin)

Berry, A.J., Copps, T., Cooper, D., Fergnson, P., Hopper, T. \& Lowe, E. A. (1985), Management Control in an Area of the NCB: Rationales of Accounting Practice in a Public Enterprise, Accounting Organizations and Society pp. 3-28.

Belkaoui, A. (1978), Linguistic Relativity in Accounting, Accounting Organizations and Society pp. 97-104.

Burchell, S., Clubb, C., Hopwood, A. \& Naphapiet,J(1980)., The Roles of Accounting in Organizations and Society, Accounting Organizations and Society pp. 5-27.

Cooper, D.J. (1980), Discussion of "Towards a Political Economy of Accounting", Accounting Organizations and Society pp. 161-166.

Cook, T. E. (1980), Political justifications: the use of standards in political appeals, The Journal of Politics pp. 511-537.

Cooper, D. J., Hayes, D. \& Wolf, F., Accounting in Organized Anarchies: Understanding and Designing Accounting Systems in Ambiguous Situations, Accounting Organizations and Society (1981) pp. 175-192.

Covaleski, M. A. \& Dirsmith, M. W. (1983), Budgeting as a Means for Control and Loose Coupling, Accounting Organizations and Society pp. 323-340.

Cherns, A. B. (1978), Alienation and Accountancy, Accounting Organizations and Society pp. $105-114$

Durkheim, E. (1957), Professional Ethics and Civic Morals (London: Routledge and Kegan Paul.

Edelman, M. (1964), The Synbolic Uses of Politics (University of Illinois Press: Urbana).

Garftnkle, H. (1967), Studies in Ethnomethodology (Englewood Cliffs, NJ: Prentice Hall).

Hopwood, A. G. (1984a), Accounting and the Pursuit of Elticiency, in Hopwood, A. G. and Tomkins, C. (eds), Issues in Public Sector Accounting (London: Philip Allen)

Holzner, B. 1968, Reality Construction in Society (Cambridge: Schenkman). 


\section{La Contabilidad Internacional: incluye visión histórica, paradigma y teorías}

Vol. 3, núm. 3., (2019)

Clara de las Mercedes Razo Ascazubi; Jeanette Lorena Moscoso Córdova; Patricia Geraldina

López Fraga

Johnson, H. T. (1980) The Search for Gain in Markets and Hierarchies: a Review of the Historical Emergence of Lehman, C. \& Tinker, T., The Gr Moving Right Show. Paper presented to the American Accounting Association, Reno, August.

Leslie, D. W., Legitimizing University Governance: Theory and Practice, Higher Education (1975) pp. 233-246.

Loft, A. (1986), Towards a critical understanding of accounting: the case of cost accounting in the U.K., 191 4-1925, Accounting Organizations and Society) () pp. 137-170

Parsons, T. (1938) The Structure of Social Action (New York: McGraw Hill,).

Parsons, T. (1968) On the concept of value - commitments, Sociologylnquiry. pp. 135-160.

Parsons, T., Social Systems and the Evolution of Action Theory (New York: Free Press, 1977).

Peckham, M. (1979) Explanation and Power. The Control of Human Behaviour (Seabury: New York,).

Shumpeter, J. (1983) American Institutions and Economic Progress, 1983, Zeitschrift fur die gesamte Staatswissenschaft Management Accounting Systems, Accounting Organizations and Society. pp. 139-146.

Mattessich, R. (1978) Instrumental Reasoning and Systems Methodology (Dordrecht/Boston: D. Reidel).

Merelman, IL M. (1966) Learning and Legitimacy, American Political Science Review pp. 548561.

Meyer, J. W. (1986) Social environments and organizational accounting, Accounting Organizations and Society pp. 345-356.

Richardson, A. J. (1987). Accounting as a legitimating institution. Accounting, Organizations and Society, 12(4), 341-355.

Richardson, A. J. (2012). Paradigms, theory and management accounting practice: A comment on Parker (forthcoming) "Qualitative management accounting research: Assessing deliverables and relevance". Critical Perspectives on Accounting, 23(1), 83-88.

Richardson, A. J. (2015). Quantitative research and the critical accounting project. Critical Perspectives on Accounting, 32, 67-77

Tiessen, P. \& Waterhouse, J. H., Towards a Descriptive Theory of Management Accounting, Accounting Organizations and Society (1983) pp. 251-267. 


\section{La Contabilidad Internacional: incluye visión histórica, paradigma y teorías}

Vol. 3, núm. 3., (2019)

Clara de las Mercedes Razo Ascazubi; Jeanette Lorena Moscoso Córdova; Patricia Geraldina López Fraga

Tinker, A. M. (1982), Merino, B. D. \& Neimark, M. D., The Normative Origins of Positive Theories: Ideology and accounting thought, Accounting Organizations and Society pp. 167200.

Thornton, D. B., Information and Institutions in the Capital Market, Accounting Organizations and Society (1979) pp. 211-234.

$$
\text { (2) (1) } \Theta(0)
$$

RECONOCIMIENTO-NOCOMERCIAL-COMPARTIRIGUAL

CC BY-NC-SA

ESTA LICENCIA PERMITE A OTROS ENTREMEZCLAR, AJUSTAR Y CONSTRUIR A PARTIR DE SU OBRA CON FINES NO

COMERCIALES, SIEMPRE Y CUANDO LE RECONOZCAN LA AUTORÍA Y SUS NUEVAS CREACIONES ESTÉN BAJO UNA LICENCIA CON LOS MISMOS TÉRMINOS. 